\title{
Evaluation of Metabolic Characteristics and Viability of Lipiodolized Hepatocellular Carcinomas Using ${ }^{18}$ F-FDG PET/CT
}

\author{
Hye Ok Kim ${ }^{1}$, Jae Seung Kim ${ }^{1}$, Yong Moon Shin ${ }^{2}$, Jin-Sook Ryu ${ }^{1}$, Yung Sang Lee ${ }^{3}$, and Seung Gyu Lee ${ }^{4}$ \\ ${ }^{1}$ Department of Nuclear Medicine, Asan Medical Center, University of Ulsan College of Medicine, Seoul, Korea; ${ }^{2}$ Department of \\ Radiology, Asan Medical Center, University of Ulsan College of Medicine, Seoul, Korea; ${ }^{3}$ Department of Internal Medicine, Asan \\ Medical Center, University of Ulsan College of Medicine, Seoul, Korea; and ${ }^{4}$ Division of Hepatobiliary Surgery and Liver \\ Transplantation, Department of Surgery, Asan Medical Center, University of Ulsan College of Medicine, Seoul, Korea
}

This study aimed to evaluate the metabolic characteristics of lipiodolized hepatocellular carcinomas (HCCs) and the diagnostic accuracy of ${ }^{18} \mathrm{~F}-\mathrm{FDG}$ PET/CT in assessing the viability of lipiodolized HCCs. Methods: Thirty-six patients (age range, 32-73 y) with 38 lipiodolized HCCs who had undergone transcatheter arterial chemoembolization (TACE) with lipiodol before ${ }^{18}$ F-FDG PET/CT (2-434 d) and 55 patients (age range, 36-77 y) with 57 treatment-naïve HCCs who had not been treated with TACE were retrospectively studied. All patients underwent hepatic lobectomy or transplantation within $1 \mathrm{mo}$ after PET/CT and multiphasic contrast-enhanced CT. ${ }^{18} \mathrm{~F}-\mathrm{FDG}$ uptake by lipiodolized and naïve HCCs was compared and correlated with tumor size, pathologic grade, serum $\alpha$-fetoprotein (AFP) concentration, and time interval between TACE and PET/CT. The diagnostic accuracy of PET/CT and contrast-enhanced CT in evaluating the viability of lipiodolized HCC was compared. Results: Histologic examination showed 30 viable and 8 nonviable lipiodolized HCCs. Of the 30 viable tumors, 19 showed increased, 10 similar, and 1 decreased ${ }^{18} \mathrm{~F}$-FDG uptake. Of the 8 nonviable HCCs, 3 showed increased and 5 decreased ${ }^{18} \mathrm{~F}-$ FDG uptake. Uptake by viable lipiodolized HCCs was correlated with tumor size $(P<0.05)$ but not correlated with pathologic grade, AFP concentration, or interval between TACE and PET/ CT. In contrast, ${ }^{18} \mathrm{~F}-\mathrm{FDG}$ uptake by naïve HCCs was significantly correlated with tumor size and pathologic grade $(P<$ 0.05 for each comparison). When lipiodolized HCCs with ${ }^{18} \mathrm{~F}$ FDG uptake that was greater than or similar to that in the surrounding normal liver were considered viable, the diagnostic sensitivity of PET/CT and contrast-enhanced CT in the early postembolic period ( $<3 \mathrm{mo}$ ) was $100 \%$ and $94 \%$, respectively, and that in the late postembolic period was $93 \%$ and $79 \%$, respectively. The specificity of ${ }^{18} \mathrm{~F}-\mathrm{FDG} \mathrm{PET} / \mathrm{CT}$ and contrastenhanced CT was $63 \%$ and $100 \%$, respectively, in the acute period. Three viable lipiodolized HCCs with high AFP concentration were true-positives on PET/CT but false-negatives on contrast-enhanced CT images. Conclusion: After TACE, ${ }^{18}$ F-FDG uptake in lipiodolized HCCs was not correlated with pathologic grade, in contrast to uptake in treatment-naïve

\footnotetext{
Received May 26, 2010; revision accepted Sep. 7, 2010.

For correspondence or reprints contact: Jae Seung Kim, Department of Nuclear Medicine, Asan Medical Center, University of Ulsan College of Medicine, Pungnap2-dong, Songpa-gu, Seoul, 138-736, Korea. E-mail: jaeskim@amc.seoul.kr

COPYRIGHT @ 2010 by the Society of Nuclear Medicine, Inc.
}

HCCs. ${ }^{18}$ F-FDG PET/CT showed a high diagnostic sensitivity in assessing the viability of lipiodolized HCCs, with moderate specificity. This method may be useful in determining the viability of lipiodolized HCCs in patients with increased serum AFP concentration or normal results on contrast-enhanced CT images.

Key Words: ${ }^{18} \mathrm{~F}-\mathrm{FDG}$; PET/CT; hepatocellular carcinoma; transcatheter arterial chemoembolization; viability

J Nucl Med 2010; 51:1849-1856

DOI: 10.2967/jnumed.110.079244

$\mathbf{H}_{\text {. }}$ epatocellular carcinoma (HCC) is one of the most common cancers worldwide (1). Liver resection and liver transplantation are the treatments of choice for patients with HCC (2). However, only approximately $20 \%$ of HCC patients are treated surgically, primarily because the tumors are often diagnosed at an advanced stage, and the associated liver cirrhosis is too well developed to permit surgical insults to be endured $(3,4)$. Palliative treatments for patients with inoperable $\mathrm{HCC}$ have included transcatheter arterial chemoembolization (TACE), systemic chemotherapy, local injection therapy, immunotherapy, and radiofrequency thermal ablation therapy (4). Although TACE is effective as either a palliative or a curative treatment for $\mathrm{HCC}$, the tumor recurrence rate after TACE is high, with reported 6- and 12-mo recurrence rates of $22.3 \%$ and $78 \%$, respectively (5). If initial treatment is insufficient, it is important to accurately evaluate tumor viability before retreatment, either by surgery or by repeated TACE.

Multiphasic contrast-enhanced CT has become the standard imaging technique in patients with HCC and has been considered the most reliable method for assessing the efficacy of TACE $(6,7)$. The ability of CT to determine tumor viability after TACE is, however, limited, because the retained hyperattenuating lipiodol material makes it difficult to detect contrast enhancement within a viable tumor $(6,7)$.

${ }^{18} \mathrm{~F}-\mathrm{FDG}$ PET is a functional imaging tool that provides metabolic information and can therefore sensitively detect both primary and metastatic tumors $(8) .{ }^{18} \mathrm{~F}-\mathrm{FDG}$ PET is of 
limited use in the diagnosis of primary HCCs, with a reported sensitivity of $50 \%-55 \%(9,10)$. This low sensitivity is attributable to high levels of glucose-6-phosphatase in HCCs, especially in well-differentiated tumors, which dephosphorylate the intracellular ${ }^{18} \mathrm{~F}$-FDG-6-phosphate $(9,11)$. Increased ${ }^{18} \mathrm{~F}$ FDG uptake by primary HCCs has been observed when tumors are large or of high grade and in patients with high serum $\alpha$-fetoprotein (AFP) concentrations (12).

Little is known about the characteristics of ${ }^{18} \mathrm{~F}-\mathrm{FDG}$ uptake and the diagnostic value of ${ }^{18} \mathrm{~F}$-FDG PET in patients with lipiodolized HCCs after TACE. ${ }^{18}$ F-FDG PET has been reported to be valuable in the assessment of tumor viability after TACE $(9,13,14)$. The cited studies, however, suffered from certain limitations: they included not only patients with $\mathrm{HCC}$ but also patients with hepatic metastatic tumors; only a few patients underwent TACE; or PET scans, and not PET/CT scans, were used. Therefore, we evaluated the metabolic characteristics of lipiodolized HCCs and the clinical ability of ${ }^{18}$ F-FDG PET/CT to assess the viability of such HCCs.

\section{MATERIALS AND METHODS}

\section{Patients}

During 3 recent years, 149 patients with HCC underwent TACE with lipiodol and ${ }^{18} \mathrm{~F}$-FDG PET/CT for preoperative tumor staging at our institution. Of these patients, 75 were treated with hepatic lobectomy or explantation for liver transplantation after PET/CT, whereas the remaining 74 patients did not undergo surgery because of extrahepatic metastases $(n=39)$, repeated TACE $(n=$ $21)$, or other reasons $(n=14)$. Of the 75 patients who underwent surgery, 36 patients (32 men, 4 women; mean age \pm SD, $54.4 \pm$ $8.3 \mathrm{y}$; range, 32-73 y) with 38 lipiodolized HCCs were retrospectively analyzed. The other 39 patients were excluded from analysis for the following reasons: a small tumor $(<2 \mathrm{~cm}$ in diameter), diabetes, an interval from PET/CT to surgery longer than $1 \mathrm{mo}$, invasive procedures performed between PET/CT and surgery, the absence of multiphasic contrast-enhanced CT at the time of PET, systemic chemotherapy before or after PET/CT, and nonvisualization of lipiodol material within the tumor.

For comparison, we assessed the metabolic characteristics of 57 HCCs in 55 consecutive treatment-naïve patients (45 men, 10 women; mean age $\pm \mathrm{SD}, 53.9 \pm 9.0 \mathrm{y}$; range, 36-78 y) who underwent PET/CT and surgery. None of these patients had small tumors $(<2 \mathrm{~cm})$, diabetes, an interval from PET/CT to surgery longer than $1 \mathrm{mo}$, invasive procedures between PET/CT and surgery, or systemic chemotherapy before or after PET/CT.

\section{TACE}

All patients underwent TACE before ${ }^{18} \mathrm{~F}-\mathrm{FDG}$ PET/CT (median, $74.0 \mathrm{~d}$ prior; range, 2-434 d). Briefly, $0.5 \mathrm{mg}$ of cisplatin (Cisplan; Dong-A Pharmaceutical Co.) per milliliter in distilled water was infused into the hepatic artery at a dose of $2 \mathrm{mg} / \mathrm{kg}$ over $15 \mathrm{~min}$. Five to ten milliliters of iodized oil (Lipiodol; Guerbert), mixed with the same volume of cisplatin, were infused into the arteries feeding the HCC. Subsequently, the selected feeding artery was embolized with absorbable gelatin sponge particles (diameter, $1 \mathrm{~mm}$; Gelfoam [Upjohn]). A 5-French 75-cm Rosch hepatic catheter and a microferret-18-infusion catheter (Cook) were used for hepatic arterial catheterization.
${ }^{18}$ F-FDG PET/CT was performed using a PET/CT camera system (Biograph Sensation 16; Siemens Medical System), which provided an axial spatial resolution of $6.3 \mathrm{~mm}$ in full width at half maximum. All patients fasted for more than $6 \mathrm{~h}$ before scanning. Whole-blood glucose concentration measured before ${ }^{18} \mathrm{~F}-\mathrm{FDG}$ administration was below $150 \mathrm{mg} / \mathrm{dL}$. Images were acquired approximately $60 \mathrm{~min}$ after intravenous injection of $555 \pm 103.6 \mathrm{MBq}$ of ${ }^{18}$ F-FDG. CT was performed in a spiral mode (110 mAs, 120 $\mathrm{kVp}$ ), from the base of the skull to the proximal thigh, for attenuation correction and image fusion, followed by a 3-dimensional caudocranial PET emission scan. The emission scan time per bed position was $2.5 \mathrm{~min}$, and 6 or 7 bed positions were used. PET data were reconstructed iteratively without and with attenuation correction based on CT data and reoriented in axial, sagittal, and coronal slices.

\section{Multiphasic Contrast-Enhanced CT}

Multiphasic contrast-enhanced CT images were obtained using a Somatom Plus-S scanner (Siemens Medical System). Nonenhanced and contrast-enhanced CT scans in the arterial (36-s delay) and portal venous (72-s delay) phases were obtained after the administration of $150 \mathrm{~mL}$ of iopamidol (Iopamiro 300; Bracco) or iopromide (Ultravist 370; Schering) at a rate of $3 \mathrm{~mL} / \mathrm{s}$. The mean interval between multiphasic contrast-enhanced CT and PET/CT was $4.1 \pm 14.3 \mathrm{~d}$.

\section{Histologic Examination}

All patients underwent hepatic lobectomy $(n=22)$ or explantation for liver transplantation $(n=14)$ within 1 mo after PET/CT. We assessed the extent of necrosis, size, and differentiation grade of each tumor from pathology reports. Necrosis was determined semiquantitatively as the percentage of necrotic tissue relative to total tumor tissue, as assessed both macroscopically and microscopically. After hematoxylin-eosin staining, each tumor was microscopically examined using low power. Totally necrotic HCCs $(100 \%)$ were defined as nonviable, and HCCs with $99 \%$ necrosis or less were defined as viable. Tumor size was determined by the longest diameter, and differentiation was graded using the Edmonson-Steiner classification (15), with grades I and II defined as low and grades III and IV as high.

\section{Data Analysis}

All PET/CT images were visually assessed by 2 experienced nuclear medicine physicians unaware of the pathologic and radiologic information. ${ }^{18} \mathrm{~F}-\mathrm{FDG}$ uptake by each $\mathrm{HCC}$ was defined as grade $\mathrm{I}$ if there was no ${ }^{18} \mathrm{~F}$-FDG uptake or if ${ }^{18} \mathrm{~F}$-FDG uptake was lower than in the surrounding normal liver, grade II if ${ }^{18} \mathrm{~F}$ FDG uptake was similar to that in the surrounding normal liver, or grade III if ${ }^{18} \mathrm{~F}$-FDG uptake was greater than that in the surrounding liver (Fig. 1). Lipiodolized HCCs scored as grade II or grade III on both attenuation-corrected and noncorrected PET images were considered to be viable.

${ }^{18} \mathrm{~F}-\mathrm{FDG}$ uptake of HCCs was analyzed according to tumor size, pathologic grade (low grade vs. high grade), serum AFP concentration ( $\leq 20 \mathrm{ng} / \mathrm{mL}$ vs. $>20 \mathrm{ng} / \mathrm{mL}$ ), presence of liver cirrhosis, and time interval between TACE and PET/CT (early postembolic period $<3$ mo vs. late period $\geq 3$ mo after TACE).

Multiphasic contrast-enhanced CT images were reviewed by a radiologist unaware of the pathologic and radiologic information. A lipiodolized HCC was considered viable when the lesion showed enhancement during the arterial phase. 

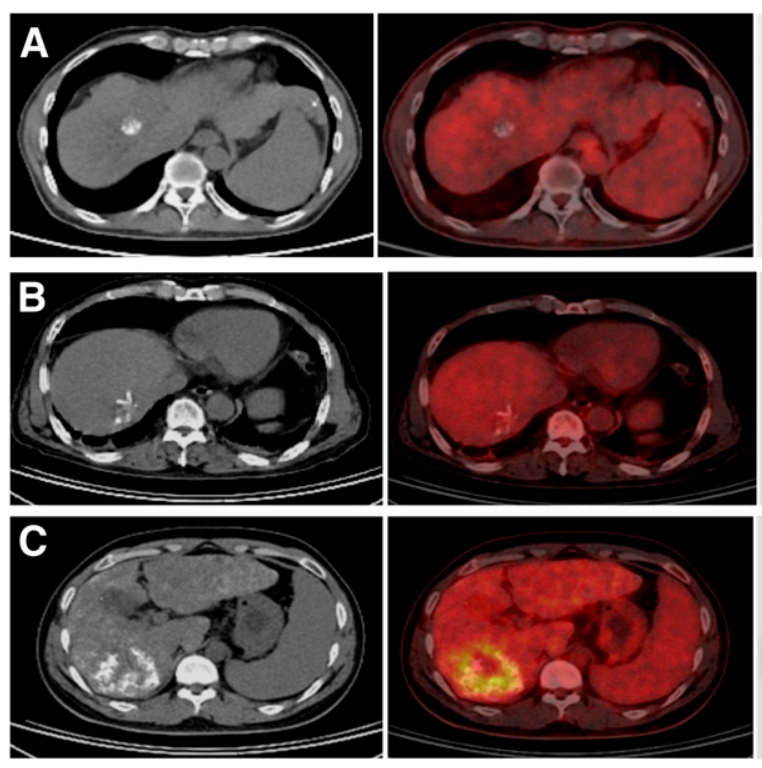
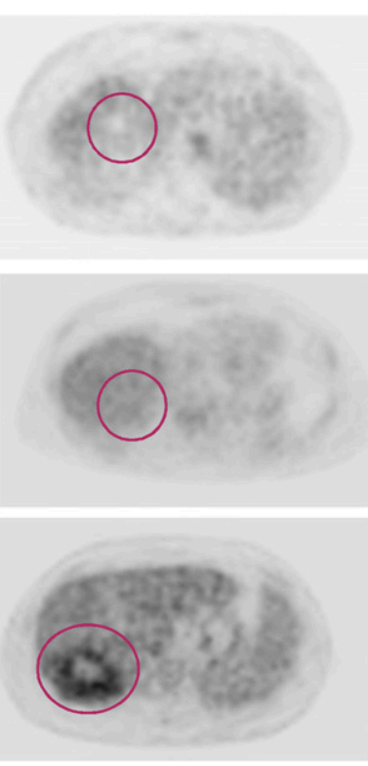

FIGURE 1. Representative images for 3-point visual grading scales of ${ }^{18} \mathrm{~F}-\mathrm{FDG}$ uptake by HCC after TACE. In tumors, grade $\mathrm{I}$ is no ${ }^{18} \mathrm{~F}-\mathrm{FDG}$ uptake or lower ${ }^{18} \mathrm{~F}-\mathrm{FDG}$ uptake than that in surrounding normal liver (A), grade II is ${ }^{18} \mathrm{~F}-\mathrm{FDG}$ uptake similar to that in surrounding normal liver (B), and grade III is higher ${ }^{18} \mathrm{~F}$-FDG uptake than that in surrounding normal liver (C). Circles indicate tumor regions.
The diagnostic sensitivity, specificity, positive predictive value, negative predictive value, and accuracy of PET/CT and multiphasic contrast-enhanced $\mathrm{CT}$ in determining the viability of lipiodolized HCCs were assessed.

\section{Statistical Analysis}

All data are expressed as mean \pm SD. Differences between lipiodolized and naïve HCC groups were analyzed using the Student $t$ test. The $\chi^{2}$ test was used to compare categoric data. A $P$ value of less than 0.05 was considered statistically significant.

\section{RESULTS}

\section{Clinical and Pathologic Characteristics}

The clinical and pathologic characteristics of lipiodolized and treatment-naïve HCCs are summarized in Table 1. The average size of lipiodolized HCCs in the longest dimension was $5.0 \pm 2.5 \mathrm{~cm}$ (range, $2-11 \mathrm{~cm}$ ). The mean interval from PET/CT to surgery was $13.3 \pm 12.6 \mathrm{~d}$, and the mean serum AFP concentration was 2,625.0 $\pm 8,279.7 \mathrm{ng} / \mathrm{mL}$. Histologically, 8 (21\%) lipiodolized HCCs showed 100\% necrosis, 1 (2\%) greater than $90 \%$ necrosis, $12(31.5 \%)$ 50-90\% necrosis, and 17 (44.7\%) less than 50\% necrosis. Thus, of the 38 lipiodolized HCCs, 30 were viable and 8 nonviable. Of the 30 viable lipiodolized HCCs, 17 $(57 \%)$ were of low grade and 13 (43\%) were of high grade.

The average size of treatment-naïve HCCs was $5.0 \pm$ $3.2 \mathrm{~cm}$ (range, $2-14 \mathrm{~cm}$ ) in the longest diameter. The mean interval from PET/CT to surgery was $10.0 \pm 7.9 \mathrm{~d}$, and the mean serum AFP concentration was 4,105.4 \pm 14,328.9

TABLE 1

Clinical and Pathologic Characteristics of Patients with HCC

\begin{tabular}{|c|c|c|c|}
\hline \multirow[b]{2}{*}{ Characteristic } & \multicolumn{2}{|c|}{$\mathrm{HCC}$} & \multirow[b]{2}{*}{$P$} \\
\hline & Lipiodolized $(n=38)$ & Naïve $(n=57)$ & \\
\hline TACE PET/CT interval (d) & & - & \\
\hline Median & 74 & & \\
\hline Range & $2-434$ & & \\
\hline PET/CT surgery interval (d) & & & NS \\
\hline Mean & 13.3 & 10.0 & \\
\hline Range & $1-31$ & $1-31$ & \\
\hline Tumor size $(\mathrm{cm})$ & $5.0 \pm 2.5$ & $5.0 \pm 3.2$ & NS \\
\hline Serum AFP level (ng/mL) & $2,625.0 \pm 8,279.7$ & $4,105.4 \pm 14,328.9$ & NS \\
\hline Liver cirrhosis & $30(79 \%)$ & $33(58 \%)$ & NS \\
\hline \multicolumn{4}{|c|}{ Pathology grade of viable HCC } \\
\hline Low $^{*}$ & $17(57 \%)$ & $41(72 \%)$ & NS \\
\hline $\mathrm{High}^{\dagger}$ & $13(43 \%)$ & $16(28 \%)$ & \\
\hline \multicolumn{4}{|c|}{$\begin{array}{l}\text { *Low grade (grade I or II) of Edmonson-Steiner classification. } \\
{ }^{*} \text { High grade (grade III or IV) of Edmonson-Steiner classification. } \\
\text { NS = not significant. }\end{array}$} \\
\hline
\end{tabular}


ng/mL. Of the 57 treatment-naïve HCCs, 41 (72\%) were classified as low grade and $16(28 \%)$ as high grade.

No significant differences were observed among patients with lipiodolized and treatment-naïve HCCs for these criteria: interval from PET/CT to surgery, tumor size, serum AFP concentration, pathologic grade, or frequency of liver cirrhosis $(P>0.05)$.

\section{Metabolic Characteristics of HCCs}

${ }^{18}$ F-FDG uptake by lipiodolized HCCs is summarized in Table 2. Of the 30 viable lipiodolized HCCs, 19 (63\%) were of grade III, $10(33 \%)$ of grade II, and $1(3 \%)$ of grade ${ }^{18} \mathrm{~F}$ FDG uptake. Of the 8 nonviable lipiodolized HCCs, 5 (63\%) were of grade I and $3(38 \%)$ of grade III ${ }^{18} \mathrm{~F}$-FDG uptake. Of the 57 naïve HCCs, 27 (47\%) were of grade III and $30(53 \%)$ of grade II ${ }^{18} \mathrm{~F}$-FDG uptake.

${ }^{18} \mathrm{~F}-\mathrm{FDG}$ uptake by viable HCCs according to various clinical factors is summarized in Table $3 .{ }^{18} \mathrm{~F}$-FDG uptake by viable lipiodolized HCCs was correlated with tumor size $(P=0.011)$. No significant differences in ${ }^{18} \mathrm{~F}-\mathrm{FDG}$ uptake according to pathologic grade, serum AFP level, or presence of liver cirrhosis regardless of the interval from TACE $(P>0.05)$ were found. However, ${ }^{18} \mathrm{~F}-\mathrm{FDG}$ uptake by naïve HCCs was greater for larger tumors $(P<0.001)$ and for tumors of high pathologic grade $(P=0.017)$.

The clinical characteristics of ${ }^{18}$ F-FDG uptake by the 8 nonviable lipiodolized HCCs are summarized in Table 4. In nonviable HCCs with grade I ${ }^{18} \mathrm{~F}$-FDG uptake $(n=5)$, the median interval from TACE was $56 \mathrm{~d}$ (range, 36-88 d), and the average tumor size was $2.8 \pm 0.6 \mathrm{~cm}$. In nonviable HCCs with grade III ${ }^{18} \mathrm{~F}$-FDG uptake $(n=3)$, the median interval from TACE to PET/CT was $39 \mathrm{~d}$ (range, 2-78 d), and the average tumor size was $4.3 \pm 2.1 \mathrm{~cm}$.

\section{Diagnostic Accuracy of Viability}

When lipiodolized HCCs with grade II or grade III ${ }^{18} \mathrm{~F}$ FDG uptake were considered viable, the overall diagnostic sensitivity and specificity of PET/CT were $97 \%$ and $63 \%$, respectively. When only lipiodolized HCCs with grade III ${ }^{18}$ F-FDG uptake were considered viable, the overall sensitivity and specificity were $63 \%$ and $63 \%$, respectively. On multiphasic contrast-enhanced CT images, 26 of the 30

TABLE 2

${ }^{18}$ F-FDG Uptake by Viable or Nonviable Lipiodolized HCC

\begin{tabular}{ccc}
\hline & \multicolumn{2}{c}{ Pathology } \\
\cline { 2 - 3 }${ }^{18}$ F-FDG uptake & Viable & Nonviable \\
\hline Grade* $^{*}$ & 1 & 5 \\
I $(n=6)$ & 10 & 0 \\
II $(n=10)$ & 19 & 3 \\
III $(n=22)$ & 30 & 8 \\
Total & &
\end{tabular}

${ }^{*}$ Grade I, no ${ }^{18} \mathrm{~F}-\mathrm{FDG}$ uptake or lower ${ }^{18} \mathrm{~F}-\mathrm{FDG}$ uptake than that in normal liver; grade II, ${ }^{8} \mathrm{~F}-\mathrm{FDG}$ uptake similar to that in normal liver; grade III, higher ${ }^{18} \mathrm{~F}-\mathrm{FDG}$ uptake than that in normal liver.

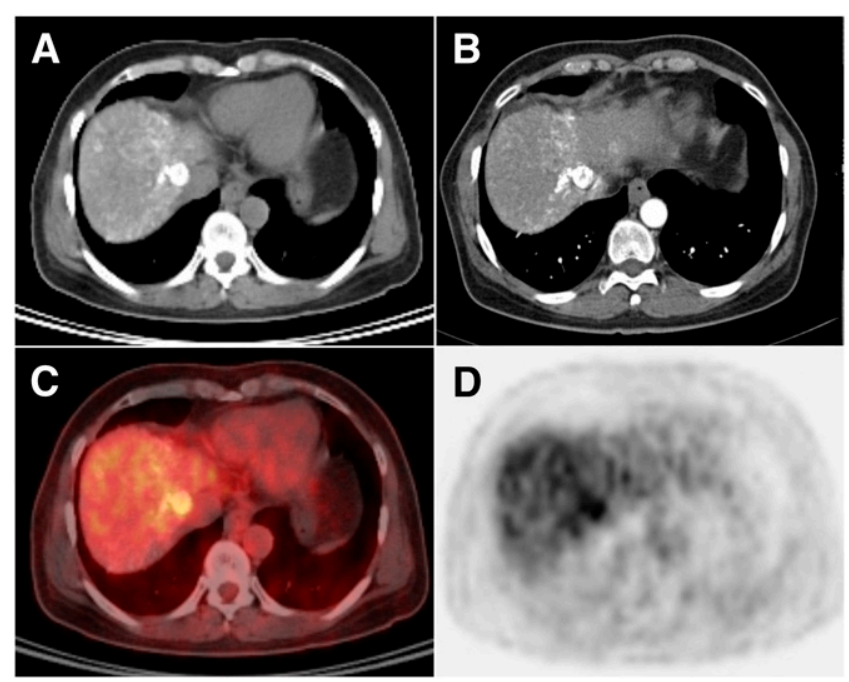

FIGURE 2. Images of 53 -y-old woman with viable (20\% necrosis) lipiodolized HCC. Interval from TACE to PET was $11 \mathrm{~d}$, and her serum AFP concentration was $156 \mathrm{ng} / \mathrm{mL}$. Multiphasic contrast-enhanced CT images (A and B) show no arterial enhancement within lipiodolized HCC. But, ${ }^{18}$ F-FDG PET/CT images (C and D) show increased (grade III) activity within tumor, compared with surrounding liver.

viable lipiodolized HCCs showed enhancement within the tumor, whereas all 8 nonviable HCCs showed no enhancement. The overall diagnostic sensitivity and specificity were $87 \%$ and $100 \%$, respectively. The diagnostic sensitivity of PET/CT and multiphasic contrast-enhanced CT in the early postembolic period was $100 \%$ and $94 \%$, respectively, and that in the late postembolic period was $93 \%$ and $79 \%$, respectively (Table 5).

Results of ${ }^{18}$ F-FDG PET/CT and multiphasic contrastenhanced CT were discordant for 6 tumors. None of these tumors showed any demonstrable viability on multiphasic contrast-enhanced CT images, whereas PET/CT images showed ${ }^{18}$ F-FDG uptake of grade II or III. Of these 6 tumors, 3-from patients with high serum AFP concentration-were viable (Fig. 2), whereas the other 3-from patients with normal AFP concentration-were nonviable histologically (Fig. 3).

On PET/CT images, only 1 tumor (size, $3.5 \mathrm{~cm} ; 85 \%$ necrosis) showed grade I ${ }^{18} \mathrm{~F}$-FDG uptake among 30 viable lipiodolized HCCs. The interval from TACE was $434 \mathrm{~d}$, and the serum AFP concentration was $2.1 \mathrm{ng} / \mathrm{mL}$. This viable tumor also showed no arterial enhancement on multiphasic contrast-enhanced CT images. Two tumors showed discrepancies in ${ }^{18} \mathrm{~F}$-FDG uptake on attenuation-corrected and noncorrected images. One showed an overcorrection artifact attributable to lipiodol, which increased the activity of the lesion on corrected but not on non-attenuation-corrected images (Fig. 4). The second tumor showed a PET/CT misregistration artifact because of respiratory movement. Both of these lipiodolized HCCs were considered nonviable on ${ }^{18}$ F-FDG PET/CT images because non-attenuation-corrected images showed lower ${ }^{18}$ F-FDG uptake than did images of the surrounding normal liver. 
TABLE 3

Characteristics of ${ }^{18}$ F-FDG Uptake in Viable Lipiodolized and Treatment-Naïve HCCs

\begin{tabular}{|c|c|c|c|c|c|c|c|c|c|c|c|c|}
\hline \multirow[b]{2}{*}{ Characteristic } & \multicolumn{3}{|c|}{$\begin{array}{c}\text { Viable lipiodolized } \\
\text { HCCs at }<3 \text { mo after } \\
\text { TACE }(n=16)\end{array}$} & \multicolumn{3}{|c|}{$\begin{array}{c}\text { Viable lipiodolized } \\
\text { HCCs at } \geq 3 \text { mo after } \\
\text { TACE }(n=14)\end{array}$} & \multicolumn{3}{|c|}{$\begin{array}{l}\text { Viable lipiodolized } \\
\text { HCCs overall } \\
(n=30)\end{array}$} & \multicolumn{3}{|c|}{$\begin{array}{c}\text { Naïve HCCs } \\
\quad(n=57)\end{array}$} \\
\hline & $\begin{array}{l}\text { Grade III } \\
\text { uptake* } \\
\left(\begin{array}{c}n=12 ; \\
75 \%)\end{array}\right.\end{array}$ & $\begin{array}{c}\text { Grade I or II } \\
\text { uptake } \\
(n=4 ; \\
25 \%)\end{array}$ & $P$ & $\begin{array}{l}\text { Grade III } \\
\text { uptake* } \\
(n=7 ; \\
50 \%)\end{array}$ & $\begin{array}{c}\text { Grade I or II } \\
\text { uptake* } \\
\begin{array}{c}(n=7 \\
50 \%)\end{array}\end{array}$ & $P$ & $\begin{array}{c}\text { Grade III } \\
\text { uptake* } \\
(n=19 ; \\
63 \%)\end{array}$ & $\begin{array}{c}\text { Grade I or II } \\
\text { uptake* } \\
(n=11 ; \\
37 \%)\end{array}$ & $P$ & $\begin{array}{l}\text { Grade III } \\
\begin{array}{c}\text { uptake* } \\
(n=27 \\
47 \%)\end{array}\end{array}$ & $\begin{array}{c}\text { Grade II } \\
\text { uptake* } \\
(n=30 ; \\
53 \%)\end{array}$ & $P$ \\
\hline $\begin{array}{l}\text { Tumor size } \\
\text { (cm) }\end{array}$ & $7.2 \pm 2.6$ & $4.1 \pm 1.4$ & 0.014 & $4.7 \pm 2.5$ & $3.7 \pm 1.4$ & NS & $6.3 \pm 1.3$ & $3.8 \pm 1.3$ & 0.011 & $6.8 \pm 3.1$ & $3.4 \pm 2.4$ & $<0.001$ \\
\hline $\begin{array}{l}\text { Pathology } \\
\text { grade }\end{array}$ & & & NS & & & NS & & & NS & & & 0.017 \\
\hline Low & 7 & 4 & & 4 & 2 & & 11 & 6 & & 15 & 26 & \\
\hline High & 5 & 0 & & 3 & 5 & & 8 & 5 & & 12 & 4 & \\
\hline AFP level & & & NS & & & NS & & & NS & & & NS \\
\hline Normal & 3 & 1 & & 3 & 5 & & 6 & 6 & & 8 & 10 & \\
\hline Increased & 9 & 3 & & 4 & 2 & & 13 & 5 & & 19 & 20 & \\
\hline Liver cirrhosis & & & NS & & & NS & & & NS & & & NS \\
\hline No & 4 & 1 & & 0 & 0 & & 4 & 1 & & 13 & 11 & \\
\hline Yes & 8 & 3 & & 7 & 7 & & 15 & 10 & & 14 & 19 & \\
\hline
\end{tabular}

${ }^{*}$ Grade I, no ${ }^{18} \mathrm{~F}-\mathrm{FDG}$ uptake or lower ${ }^{18} \mathrm{~F}-\mathrm{FDG}$ uptake than that in normal liver; grade II, ${ }^{18} \mathrm{~F}-\mathrm{FDG}$ uptake similar to that in normal liver; grade III, higher ${ }^{18} \mathrm{~F}-\mathrm{FDG}$ uptake than that in normal liver.

NS = not significant.

\section{DISCUSSION}

HCCs have been shown to accumulate ${ }^{18}$ F-FDG to varying degrees, with the size and degree of differentiation of the tumor and serum AFP concentration being important predictors of tumor visualization by ${ }^{18} \mathrm{~F}$-FDG PET. We found that $65 \%$ of low-grade and $62 \%$ of high-grade viable lipiodolized HCCs, compared with $37 \%$ of low-grade and $75 \%$ of high-grade treatment-naïve HCCs, showed increased ${ }^{18}$ F-FDG uptake. In addition, lipiodolized HCCs showed no difference in ${ }^{18} \mathrm{~F}$-FDG uptake with respect to serum AFP concentration or other clinical factors, except

TABLE 4

Clinical Characteristics of Nonviable Lipiodolized HCCs

\begin{tabular}{ccc}
\hline Characteristic & $\begin{array}{c}\text { False-positive } \\
(n=3) \text { result } \\
\text { on PET/CT }\end{array}$ & $\begin{array}{c}\text { True-negative } \\
(n=5) \text { result } \\
\text { on PET/CT }\end{array}$ \\
\hline 18F-FDG uptake & Grade III & Grade I \\
TACE PET interval (d) & & \\
Median & 39 & 56 \\
Range & $2-78$ & $36-88$ \\
Size $(\mathrm{cm})$ & $4.3 \pm 2.1$ & $2.8 \pm 0.6$ \\
Location in liver & & \\
Central & 1 & 3 \\
Peripheral & 2 & 2 \\
Glucose level (mg/dL) & $110.3 \pm 28.7$ & $100.6 \pm 8.3$ \\
Liver cirrhosis & $2(67 \%)$ & $3(60 \%)$
\end{tabular}

${ }^{*}$ Grade I, no ${ }^{18} \mathrm{~F}-\mathrm{FDG}$ uptake or lower ${ }^{18} \mathrm{~F}-\mathrm{FDG}$ uptake than that in normal liver; grade III, higher ${ }^{18} \mathrm{~F}-\mathrm{FDG}$ uptake than that in normal liver. tumor size. Previous studies have reported that only 50\%$55 \%$ of primary HCCs, compared with normal liver parenchyma, show increased ${ }^{18} \mathrm{~F}$-FDG uptake $(9,10)$. The relatively low sensitivity of ${ }^{18} \mathrm{~F}$-FDG PET in the detection of HCC may be attributed to high levels of glucose-6-phosphatase, especially in low-grade and well-differentiated HCCs that show low expression of glucose transporter $1(9,11,16,17)$. High levels of glucose-6-phosphatase cause dephosphorylation of ${ }^{18} \mathrm{~F}$-FDG-6-phosphate and leakage of ${ }^{18} \mathrm{~F}-\mathrm{FDG}$ back to the circulation. As the HCC stage becomes more advanced, lesions tend to become undifferentiated (18). Our findings were similar, in that the grade and size of treatment-naïve $\mathrm{HCCs}$ correlated with ${ }^{18} \mathrm{~F}$-FDG uptake. Although Risse et al. reported that HCCs were not correlated with the degree of differentiation (19), this study included selected patients for ${ }^{131}$ I-lipiodol treatment and only 2 high-grade tumors. Thus, this interstudy difference from our study could affect the controversial result. Interestingly, however, we found that, in contrast to treatmentnaïve HCCs, lipiodolized HCCs-particularly low-grade tumors-frequently showed increased ${ }^{18}$ F-FDG uptake. This may be caused by increased glycolysis in residual tumors after TACE. HCCs pretreated with TACE displayed significantly higher hexokinase II messenger RNA expression, and there was a significant correlation between the expression of hypoxia-inducible factor $1 \alpha(\mathrm{HIF}-1 \alpha)$ protein and hexokinase II messenger RNA (20). Furthermore, both HIF- $1 \alpha$ and hexokinase II protein were colocalized in cancer cells near necrotic regions. HIF- $1 \alpha$ expression was observed only after TACE upregulation of hexokinase II, allowing cancer cells to obtain sufficient energy for growth 
TABLE 5

Diagnostic Accuracy of PET/CT and Multiphasic Contrast-Enhanced CT in Assessing Viability of Lipiodolized HCC

\begin{tabular}{|c|c|c|c|c|c|c|c|}
\hline Modality & $\begin{array}{l}\text { Positive criteria for } \\
\text { viability }\end{array}$ & $\begin{array}{l}\text { Interval after } \\
\text { TACE }\end{array}$ & $\begin{array}{l}\text { Sensitivity } \\
\text { (\%) }\end{array}$ & $\begin{array}{l}\text { Specificity } \\
(\%)\end{array}$ & $\begin{array}{l}\text { Positive predictive } \\
\text { value (\%) }\end{array}$ & $\begin{array}{c}\text { Negative predictive } \\
\text { value (\%) }\end{array}$ & $\begin{array}{c}\text { Accuracy } \\
\text { (\%) }\end{array}$ \\
\hline \multirow[t]{6}{*}{ PET } & Grade II or III ${ }^{18} \mathrm{~F}-\mathrm{FDG}$ & Overall & 97 & 63 & 91 & 83 & 89 \\
\hline & uptake* & Acute $^{\dagger}$ & 100 & 63 & 84 & 100 & 88 \\
\hline & & Chronic $^{\ddagger}$ & 93 & $N A^{9)}$ & 100 & 0 & 93 \\
\hline & Grade III ${ }^{18} \mathrm{~F}-\mathrm{FDG}$ uptake & Overall & 63 & 63 & 86 & 31 & 63 \\
\hline & only ${ }^{\star}$ & Acute $^{\dagger}$ & 75 & 63 & 80 & 56 & 71 \\
\hline & & Chronic $\ddagger$ & 50 & NA & 100 & 0 & 50 \\
\hline \multirow[t]{3}{*}{ CT } & Arterial enhancement & Overall & 87 & 100 & 100 & 67 & 89 \\
\hline & & Acute $^{\dagger}$ & 94 & 100 & 100 & 89 & 96 \\
\hline & & Chronic ${ }^{\ddagger}$ & 79 & NA & 100 & 0 & 79 \\
\hline
\end{tabular}

${ }^{*}$ Grade II, ${ }^{18} \mathrm{~F}-\mathrm{FDG}$ uptake similar to that in normal liver; grade III, higher ${ }^{18} \mathrm{~F}-\mathrm{FDG}$ uptake than that in normal liver.

${ }^{\dagger}$ Acute, $<3$ mo after TACE.

${ }^{\ddagger}$ Chronic, $\geq 3$ mo after TACE.

$\mathrm{NA}=$ not applicable.

under the condition of acute hypoxia caused by TACE. During the abrupt devascularization caused by TACE, HIF-1 $\alpha$ expressed in HCCs tends to induce hexokinase II expression, mediating a phenotypic change enabling cells to adapt to a hypoxic environment.

The increased ${ }^{18} \mathrm{~F}$-FDG uptake observed in lipiodolized, compared with naïve, tumors may be also attributable to a more aggressive growth of residual viable tumor when TACE is not completely effective. TACE has been shown to stimulate tumor angiogenesis, thus increasing the proliferative activity of tumor cells to some degree (21-25). After TACE, tumor cells are exposed to an extremely hypoxic or even anoxic environment. Because of ischemic damage,
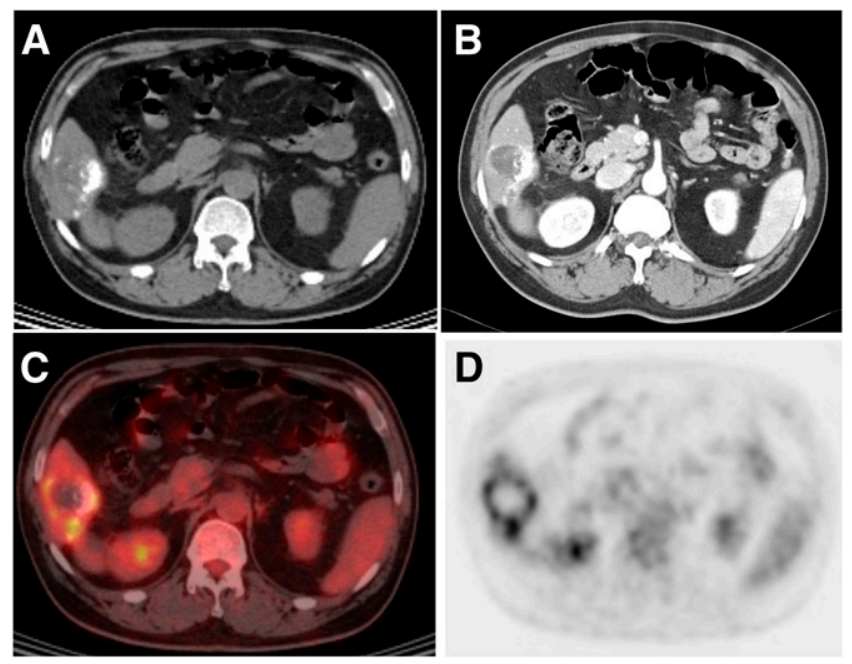

FIGURE 3. Images of 55-y-old man with nonviable lipiodolized HCC. Interval from TACE to PET was $78 \mathrm{~d}$, and his serum AFP concentration was $5.8 \mathrm{ng} / \mathrm{mL}$. Multiphasic contrast-enhanced CT images (A and $B$ ) show no arterial enhancement within lipiodolized HCC. ${ }^{18}$ F-FDG PET/CT images (C and D) show increased (grade III) activity in marginal portion of tumor, compared with surrounding liver. vascular endothelial growth factor and basic fibroblast growth factor, which act synergistically, are overexpressed after TACE $(21,23)$. Levels of these angiogenic factors were found to be significant predictors of clinical outcomes in patients with HCC $(26,27)$. The long-term survival of HCC patients after TACE remains unsatisfactory (28), and the 18-mo recurrence rate after TACE has been reported to be higher than that for untreated patients (5). Thus, the increase in ${ }^{18} \mathrm{~F}-\mathrm{FDG}$ uptake after TACE may be caused by increased glycolysis and the aggressive behavior of any residual viable tumor.

Patients with HCCs detectable by ${ }^{18}$ F-FDG PET have been found to have significantly higher serum AFP concentrations than do patients with undetectable HCCs (12). Serum AFP concentration has been found to correlate significantly with standardized uptake value (SUV) or the tumor-to-normal ratio, indicating that AFP is involved in glucose metabolism and cell proliferation in HCC (29). We found, however, that ${ }^{18} \mathrm{~F}$-FDG uptake was not correlated with serum AFP concentration, in patients with either lipiodolized HCCs or treatment-naïve HCCs. No correlation of AFP concentration and ${ }^{18} \mathrm{~F}-\mathrm{FDG}$ uptake in our study might be attributable to the interstudy differences in patient population. It has been known that serum AFP values are frequently elevated in patients with chronic liver disease, even in the absence of HCC. In addition, many factors associated with raised AFP include the etiology and severity of liver disease, sex, and race (30). In contrast to the previous studies $(12,29)$, our study population consisted of surgically resectable patients without distant metastasis, a high proportion of patients with high-grade tumors, and a relatively high proportion of patients with hepatitis B virus infections.

In our analysis, we regarded lipiodolized $\mathrm{HCCs}$ with ${ }^{18} \mathrm{~F}-$ FDG uptake either greater than or similar to that in the surrounding normal liver tissue as viable. These tumors were considered viable because PET/CT was able to iden- 


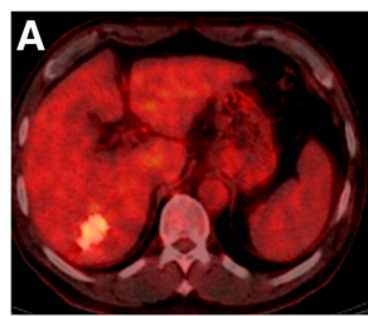

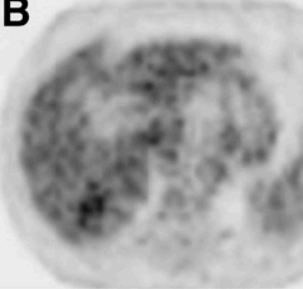

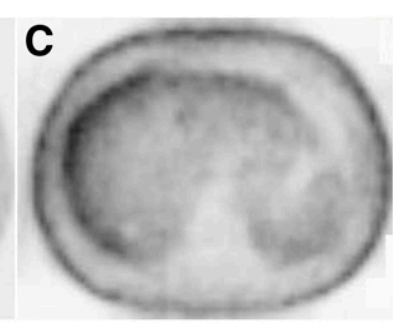

FIGURE 4. Images of 51-y-old man with nonviable lipiodolized HCC. Attenuation-corrected ${ }^{18} \mathrm{~F}-\mathrm{FDG} \mathrm{PET} / \mathrm{CT}$ images ( $\mathrm{A}$ and $\mathrm{B}$ ) show increased (grade III) activity of lipiodolized HCC, but noncorrected ${ }^{18} \mathrm{~F}-\mathrm{FDG}$ PET/CT image (C) shows decreased (grade I) activity, compared with surrounding liver. Increased activity of lipiodolized HCC observed on attenuation-corrected images is likely caused by attenuation-correction artifact. tify the margins of lipiodolized HCCs, and necrotic tissue, compared with normal liver, usually shows significantly decreased ${ }^{18}$ F-FDG uptake (11). Of the 30 viable lipiodolized HCCs, 29 (97\%) showed ${ }^{18}$ F-FDG uptake higher than or similar to that in the surrounding normal liver tissue and could therefore be correctly diagnosed as viable on PET/ CT. However, 26 of these 30 viable lipiodolized HCCs showed enhancement on multiphasic contrast-enhanced CT. In comparing the results of ${ }^{18} \mathrm{~F}-\mathrm{FDG} \mathrm{PET} / \mathrm{CT}$ with multiphasic contrast-enhanced CT, we found that 6 tumors yielded discordant results. PET/CT correctly diagnosed 3 viable HCCs in patients with increased AFP levels, whereas these 3 patients were false-negatives on multiphasic contrast-enhanced CT. The other 3 nonviable HCCs in patients with normal AFP levels were true-negatives on multiphasic contrast-enhanced CT. Torizuka et al. reported that increased or similar ${ }^{18} \mathrm{~F}-\mathrm{FDG}$ uptake by a tumor with respect to surrounding normal tissue suggested the presence of residual viable tumor tissue, whereas decreased or absent ${ }^{18} \mathrm{~F}$-FDG uptake indicated greater than $90 \%$ necrosis, showing that therapy had been effective (14). In addition, these PET findings appeared to reflect tumor viability more accurately than did intratumor lipiodol retention on $\mathrm{CT}$. Chen et al. reported that ${ }^{18}$ F-FDG PET detected 20 of $30(67 \%)$ recurrent HCCs in patients showing increasing AFP concentrations after surgical resection or interventional therapy, when results of conventional examinations were normal (31). These findings suggest that ${ }^{18} \mathrm{~F}-\mathrm{FDG}$ PET/ CT may be useful for detecting viable lipiodolized HCCs in patients with increased serum AFP but in whom no demonstrable viability is seen on multiphasic contrastenhanced CT images.

After TACE, HCCs undergo ischemic necrosis, and liver tissue surrounding the HCC may become inflamed, especially during the early postembolic period (32). ${ }^{18} \mathrm{~F}-\mathrm{FDG}$ accumulates in inflammatory cells such as neutrophils, lymphocytes, and macrophages (33). Of the 8 nonviable HCCs studied in the present report, 3 showed increased ${ }^{18} \mathrm{~F}-\mathrm{FDG}$ uptake (false-positives). Because of the retrospective design of our study, how much inflammatory cells were infiltrated in false-positive cases could not be confirmed. However, because all of our false-positive cases were observed in the early postembolic period and $75 \%$ of viable HCCs in the early postembolic period showed increased ${ }^{18} \mathrm{~F}-\mathrm{FDG}$ uptake regardless of pathologic grade, it was determined that inflammatory cells may contribute to the overall ${ }^{18} \mathrm{~F}$ FDG uptake in nonviable lipiodolized HCCs, but also viable tumors, within 3 mo after TACE.

The presence of highly attenuating materials, such as metal and lipiodol (34), during ${ }^{18} \mathrm{~F}-\mathrm{FDG}$ PET/CT may result in artifacts. If lipiodol affects ${ }^{18} \mathrm{~F}-\mathrm{FDG}$ uptake, the error must be present more or less in lipiodolized HCCs. Uncorrected images may be helpful if the lesions are located in the periphery of the liver but not for central lesions. Moreover, respiratory motion artifacts at the interface between the lungs and diaphragm may be present on CT-attenuation-corrected PET images. These artifacts may be caused by undercorrection of attenuation of the upper liver, because lung volumes are larger on CT scans than on emission PET images $(35,36)$. Therefore, we evaluated PET/CT images, including PET images that were not corrected for attenuation, to detect any artificially increased activity. We observed 2 lipiodolized HCCs with falsely elevated activity on attenuation-corrected, compared with noncorrected, PET/CT images, suggesting the importance of evaluating noncorrected images in the detection of viable lipiodolized HCCs.

Our study had several limitations. First, the retrospective design may introduce biases in the diagnostic accuracy of ${ }^{18} \mathrm{~F}$ FDG PET/CT and multiphasic contrast-enhanced CT. Moreover, we included only patients who underwent surgery after ${ }^{18}$ F-FDG PET/CT for the determination of pathologic correlations. Thus, the relatively small number of nonviable HCCs may have affected the diagnostic specificity data. Second, we did not quantitatively measure ${ }^{18}$ F-FDG uptake. Although SUV is the most commonly used quantitative measurement of ${ }^{18}$ F-FDG uptake, SUV can be influenced by factors other than glucose metabolism and there is no absolute threshold for determining tumor viability. Because viable HCCs can be detected by contrast between the tumor and the surrounding liver, we evaluated HCC uptake of ${ }^{18}$ F-FDG by semiquantitative visual comparison with adjacent normal tissue. Increased or similar tumor ${ }^{18} \mathrm{~F}$-FDG uptake, compared with that in surrounding normal liver, as measured by the SUV ratio, has been reported to indicate tumor viability after interventional therapy (14). Third, we defined HCCs with less than $99 \%$ necrosis on histology as viable. Because a small lipiodolized HCC with more than $90 \%$ necrosis may show decreased ${ }^{18} \mathrm{~F}$-FDG uptake despite the presence of residual viable tumor, as a result of a partial-volume effect, care should be taken in evaluating such 
small tumors. However, in our study, there was only 1 lipiodolized HCC with more than $90 \%$ necrosis, which showed increased ${ }^{18} \mathrm{~F}$-FDG uptake.

\section{CONCLUSION}

After TACE, ${ }^{18} \mathrm{~F}-\mathrm{FDG}$ uptake was not correlated with pathologic grade, in contrast to treatment-naïve HCCs. ${ }^{18} \mathrm{~F}$ FDG PET/CT showed high diagnostic sensitivity in detecting viable HCCs, with moderate specificity. This method may be useful in determining the viability of lipiodolized HCCs in patients with increased serum AFP concentration or normal results on multiphasic contrast-enhanced CT.

\section{REFERENCES}

1. Schafer DF, Sorrell MF. Hepatocellular carcinoma. Lancet. 1999;353:1253-1257.

2. Wall WJ, Marotta PJ. Surgery and transplantation for hepatocellular cancer. Liver Transpl. 2000;6:S16-S22.

3. Nagorney DM, van Heerden JA, Ilstrup DM, Adson MA. Primary hepatic malignancy: surgical management and determinants of survival. Surgery. 1989;106: 740-748.

4. Lee JK, Chung YH, Song BC, et al. Recurrences of hepatocellular carcinoma following initial remission by transcatheter arterial chemoembolization. $J$ Gastroenterol Hepatol. 2002;17:52-58.

5. Cheng HY, Wang X, Chen D, Xu AM, Jia YC. The value and limitation of transcatheter arterial chemoembolization in preventing recurrence of resected hepatocellular carcinoma. World J Gastroenterol. 2005;11:3644-3646.

6. Takayasu K, Arii S, Matsuo N, et al. Comparison of CT findings with resected specimens after chemoembolization with iodized oil for hepatocellular carcinoma. AJR. 2000;175:699-704.

7. Guan YS, Sun L, Zhou XP, Li X, Zheng XH. Hepatocellular carcinoma treated with interventional procedures: CT and MRI follow-up. World J Gastroenterol. 2004; 10:3543-3548.

8. Strauss LG, Conti PS. The applications of PET in clinical oncology. J Nucl Med. 1991;32:623-648.

9. Okazumi S, Isono K, Enomoto K, et al. Evaluation of liver tumors using fluorine18-fluorodeoxyglucose PET: characterization of tumor and assessment of effect of treatment. J Nucl Med. 1992;33:333-339.

10. Khan MA, Combs CS, Brunt EM, et al. Positron emission tomography scanning in the evaluation of hepatocellular carcinoma. J Hepatol. 2000;32:792-797.

11. Torizuka T, Tamaki N, Inokuma T, et al. In vivo assessment of glucose metabolism in hepatocellular carcinoma with FDG-PET. J Nucl Med. 1995;36:1811-1817.

12. Trojan J, Schroeder O, Raedle J, et al. Fluorine-18 FDG positron emission tomography for imaging of hepatocellular carcinoma. Am J Gastroenterol. 1999; 94:3314-3319.

13. Vitola JV, Delbeke D, Meranze SG, Mazer MJ, Pinson CW. Positron emission tomography with F-18-fluorodeoxyglucose to evaluate the results of hepatic chemoembolization. Cancer. 1996;78:2216-2222.

14. Torizuka T, Tamaki N, Inokuma T, et al. Value of fluorine-18-FDG-PET to monitor hepatocellular carcinoma after interventional therapy. J Nucl Med. 1994;35:1965-1969.

15. Edmondson HA, Steiner PE. Primary carcinoma of the liver: a study of 100 cases among 48,900 necropsies. Cancer. 1954;7:462-503.

16. Roh MS, Jeong JS, Kim YH, Kim MC, Hong SH. Diagnostic utility of GLUT1 in the differential diagnosis of liver carcinomas. Hepatogastroenterology. 2004;51: 1315-1318.
17. Zimmerman RL, Burke M, Young NA, Solomides CC, Bibbo M. Diagnostic utility of Glut-1 and CA 15-3 in discriminating adenocarcinoma from hepatocellular carcinoma in liver tumors biopsied by fine-needle aspiration. Cancer. 2002;96: 53-57.

18. Sasaki Y, Imaoka S, Ishiguro S, et al. Clinical features of small hepatocellular carcinomas as assessed by histologic grades. Surgery. 1996;119:252-260.

19. Risse JH, Pauleit D, Bender H, et al. ${ }^{18}$ F-FDG-PET and histopathology in ${ }^{131} \mathrm{I}-$ lipiodol treatment for primary liver cancer. Cancer Biother Radiopharm. 2009; 24:445-452.

20. Yasuda S, Arii S, Mori A, et al. Hexokinase II and VEGF expression in liver tumors: correlation with hypoxia-inducible factor 1 alpha and its significance. J Hepatol. 2004;40:117-123.

21. von Marschall Z, Cramer T, Hocker M, Finkenzeller G, Wiedenmann B, Rosewicz S. Dual mechanism of vascular endothelial growth factor upregulation by hypoxia in human hepatocellular carcinoma. Gut. 2001;48:87-96.

22. Wang B, Xu H, Gao ZQ, Ning HF, Sun YQ, Cao GW. Increased expression of vascular endothelial growth factor in hepatocellular carcinoma after transcatheter arterial chemoembolization. Acta Radiol. 2008;49:523-529.

23. Li X, Feng GS, Zheng CS, Zhuo CK, Liu X. Influence of transarterial chemoembolization on angiogenesis and expression of vascular endothelial growth factor and basic fibroblast growth factor in rat with Walker-256 transplanted hepatoma: an experimental study. World J Gastroenterol. 2003;9:2445-2449.

24. Sergio A, Cristofori C, Cardin R, et al. Transcatheter arterial chemoembolization (TACE) in hepatocellular carcinoma (HCC): the role of angiogenesis and invasiveness. Am J Gastroenterol. 2008;103:914-921.

25. Shim JH, Park JW, Kim JH, et al. Association between increment of serum VEGF level and prognosis after transcatheter arterial chemoembolization in hepatocellular carcinoma patients. Cancer Sci. 2008;99:2037-2044.

26. Chao Y, Li CP, Chau GY, et al. Prognostic significance of vascular endothelial growth factor, basic fibroblast growth factor, and angiogenin in patients with resectable hepatocellular carcinoma after surgery. Ann Surg Oncol. 2003;10:355-362.

27. Mise M, Arii S, Higashituji H, et al. Clinical significance of vascular endothelial growth factor and basic fibroblast growth factor gene expression in liver tumor. Hepatology. 1996;23:455-464.

28. Farinati F, De Maria N, Marafin C, et al. Unresectable hepatocellular carcinoma in cirrhosis: survival, prognostic factors, and unexpected side effects after transcatheter arterial chemoembolization. Dig Dis Sci. 1996;41:2332-2339.

29. Shang JB, Li YH, Liu FY, et al. ${ }^{18}$ F-Fluorodeoxyglucose uptake in hepatocellular carcinoma on positron emission tomography correlates with alpha-fetoprotein. Di Yi Jun Yi Da Xue Xue Bao. 2004;24:697-699.

30. Di Bisceglie AM, Sterling RK, Chung RT, et al. Serum alpha-fetoprotein levels in patients with advanced hepatitis C: results from the HALT-C Trial. J Hepatol. 2005;43:434-441.

31. Chen YK, Hsieh DS, Liao CS, et al. Utility of FDG-PET for investigating unexplained serum AFP elevation in patients with suspected hepatocellular carcinoma recurrence. Anticancer Res. 2005;25:4719-4725.

32. Jiang B, Lou Q, Ding XF, et al. Histopathological changes in rat transplanted hepatoma after lipiodol transarterial embolization. Zhonghua Zhong Liu Za Zhi. 2004;26:205-208.

33. Meller J, Sahlmann C-O, Scheel AK. ${ }^{18}$ F-FDG PET and PET/CT in fever of unknown origin. J Nucl Med. 2007;48:35-45.

34. Heusner TA, Fronz U, Jentzen W, et al. The effect of different chemoembolization materials on CT-based attenuation correction in PET/CT [in German]. Rofo. 2007;179:1159-1165.

35. Osman MM, Cohade C, Nakamoto Y, Wahl RL. Respiratory motion artifacts on PET emission images obtained using CT attenuation correction on PET-CT. Eur J Nucl Med Mol Imaging. 2003;30:603-606.

36. Papathanassiou D, Becker S, Amir R, Meneroux B, Liehn JC. Respiratory motion artefact in the liver dome on FDG PET/CT: comparison of attenuation correction with CT and a caesium external source. Eur J Nucl Med Mol Imaging. 2005;32:1422-1428. 